\title{
Comparison of Magnesium II core-to-wing ratio observations during solar minimum $23 / 24$
}

\author{
Martin Snow ${ }^{1, *}$, Mark Weber ${ }^{2}$, Janet Machol ${ }^{3,4}$, Rodney Viereck ${ }^{5}$, and Erik Richard ${ }^{1}$ \\ 1 Laboratory for Atmospheric and Space Physics (LASP), University of Colorado, Boulder, CO 80309, USA \\ *Corresponding author: e-mail: snow@lasp.colorado.edu \\ 2 Institute of Environmental Physics, University of Bremen FB1, PO Box 330440, 28334 Bremen, Germany \\ 3 Cooperative Institute for Research in Environmental Sciences (CIRES), University of Colorado, Boulder, CO 80309, USA \\ 4 National Geophysical Data Center (NGDC), National Oceanic and Atmospheric Administration, Boulder, CO 80305, USA \\ 5 Space Weather Prediction Center (SWPC), National Oceanic and Atmospheric Administration, Boulder, CO 80305, USA
}

Received 15 September 2013 / Accepted 5 December 2013

\begin{abstract}
The MgII index is an important proxy for solar activity; in particular it correlates well with the EUV which is important for understanding the upper atmosphere. We compare the measurements from all of the instruments making daily measurements during the most recent solar minimum. After adjustments to the data to account for instrumental effects, we find that there are still some discrepancies between the various time series. The data from the primary channel of the SOLar-STellar Irradiance Comparison Experiment (SOLSTICE) on SORCE requires a correction factor starting in early 2006 in order to bring it into agreement with the redundant SOLSTICE channel and with the other datasets. Once this correction has been applied, all the MgII measurements are in good agreement throughout the solar minimum interval.
\end{abstract}

Key words. solar activity - UV flux - solar cycle

\section{Introduction}

The primary energy input to the upper atmosphere of the Earth is extreme ultraviolet (EUV; $10-121 \mathrm{~nm}$ ) radiation from the Sun. Variations in the solar EUV flux drive the chemistry and dynamics of the thermosphere and ionosphere. EUV photons are absorbed by $\mathrm{O}, \mathrm{O}_{2}$, and $\mathrm{N}_{2}$ in the atmosphere above $100 \mathrm{~km}$, heating it. Ionization of the neutral atmosphere by the EUV flux on the daylight side of the Earth creates the ionosphere. Over the eleven year solar cycle, the EUV irradiance changes by a factor of 2-5 depending on wavelength (Lean et al. 2011). Models of the atmosphere such as the Thermosphere Ionosphere Mesosphere Electrodynamic General Circulation Model (TIMEGCM; Solomon \& Qian 2005) all need solar EUV flux as an input. There are currently direct daily measurements of the solar EUV flux, but this has not always been the case. Atmospheric modelers have had to rely on a more commonly available measurement which can be used to represent the true EUV irradiance.

Heath \& Schlesinger (1986) identified the Magnesium II core-to-wing ratio as a useful proxy for the solar EUV irradiance. This ratio is also known as the MgII index and for a low-resolution $(1.1 \mathrm{~nm})$ instrument is defined as:

$$
I=\frac{4\left[E_{279.8}+E_{280.0}+E_{280.2}\right]}{3\left[E_{276.6}+E_{276.8}+E_{283.2}+E_{283.4}\right]},
$$

where $E_{\lambda}$ is the spectral irradiance measured at wavelength $\lambda$ and $I$ is the MgII index. Higher resolution instruments $(<0.3 \mathrm{~nm})$ often use a different algorithm for determining the numerator of the ratio, but in all cases the MgII index is a ratio of the chromospheric contribution to the photospheric contribution.
The MgII index is fairly easy to measure and is relatively insensitive to instrumental artifacts. Of course, it can be a challenge to turn those qualitative statements into quantitative datasets. In particular, the spectral resolution of the instrument making the observation and the sampling of the spectrum can have an impact on the precision of the MgII index measurement (Snow \& McClintock 2005; Snow et al. 2005).

The time range considered in this paper is the decline of solar cycle 23 , through the long solar minimum, and into the rise of cycle 24 (2000-2013). Figure 1 shows operating lifetimes for each of the missions that measured the MgII index during this time period. At any one time, there are multiple instruments taking daily measurements. The goal of this paper is to combine these observations into a single consistent time series that best represents the solar activity. A future paper will link these observations to the prior composite data record described in Viereck et al. (2004). That composite covered the years 1978 through 2003. Ultimately, we will have a single consistent MgII time series from 1978 through the present covering more than 35 years.

\section{Measurements of the Mgll index during solar minimum}

During solar minimum 23/24, the number of instruments measuring the MgII index was larger than it has ever been. Having a wealth of observations is always a good thing, but understanding the differences between the various datasets can be non-trivial. In this section, we catalog the available observations. Our study is limited to those instruments which observe the appropriate region of the solar spectrum on a daily basis and 


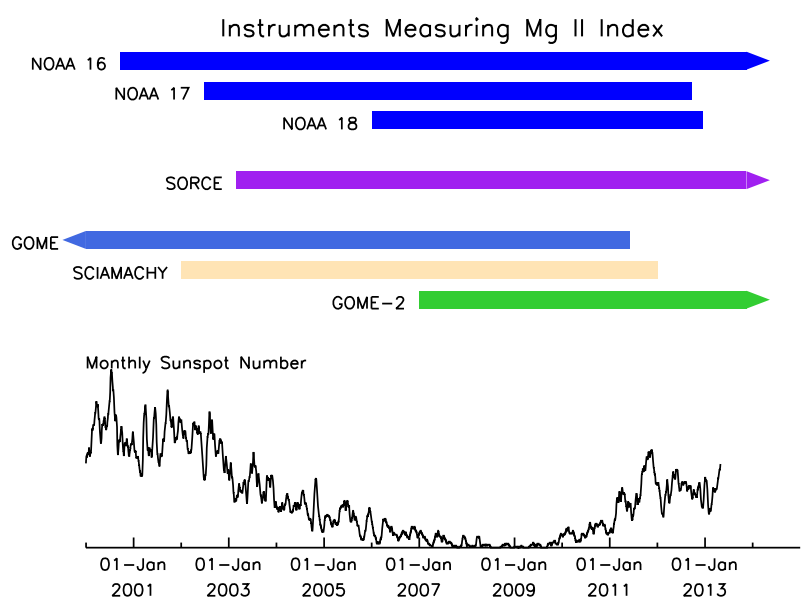

Fig. 1. MgII index datasets discussed in this paper. The top three blue bars are the Solar Backscatter Ultraviolet (SBUV/2) instruments on NOAA 16, 17, and 18. The purple bar shows the time range covered by SORCE SOLSTICE. The bottom three bars (light blue, peach, and green) show the observational time coverage for GOME, SCIAMACHY, and GOME-2 respectively. An arrowhead on a colored bar indicates that the dataset continues to the present day, or in the case of GOME, it indicates that the dataset begins before the earliest date on the plot.

release the data to the public. All the observations used here come from satellites in low Earth orbit.

\subsection{NOAA}

The National Oceanic and Atmospheric Administration (NOAA) has operated a series of instruments which measure the solar irradiance near $280 \mathrm{~nm}$ dating back to 1978 . For the time period of this study, there were three Solar Backscattered UltraViolet version $2(\mathrm{SBUV} / 2)$ instruments in service on NOAA 16-18. The design of these instruments is described in Frederick et al. (1986). A fourth instrument on the NOAA 19 satellite went into operation part way through solar minimum, but data from this instrument have not yet been made available to the public, so we do not include it in our analysis.

MgII measurements from the SBUV/2 series began with NOAA 9, and were first described in Cebula et al. (1992). The SBUV/2 instrument can measure the solar spectrum in either a "sweep" mode where the detector is read while the grating scans in a continuous fashion, or with a "discrete" mode where the grating pauses at pre-defined angles and the detector is only sampled during these pauses. The "discrete" mode data is described in Donnelly $(1988,1990)$, and a comparison of the two methods is described in DeLand \& Cebula (1993). The NOAA missions described here take just one solar measurement per day.

The solar irradiance spectrum observed by an SBUV/2 instrument has a spectral resolution of $1.1 \mathrm{~nm}$ and looks very similar to the dashed line in Figure 2. In addition to the four marked wing irradiance values (black dots), the MgII index from SBUV/2 uses three measurements in the core of the line $(279.8,280.0$, and $280.2 \mathrm{~nm})$ as described by Heath \& Schlesinger (1986). Data files of the SBUV/2 MgII index measurements used in this study were retrieved from http:// sbuv2.gsfc.nasa.gov/solar/mgii/. These data products are all derived from the "discrete" mode observations.

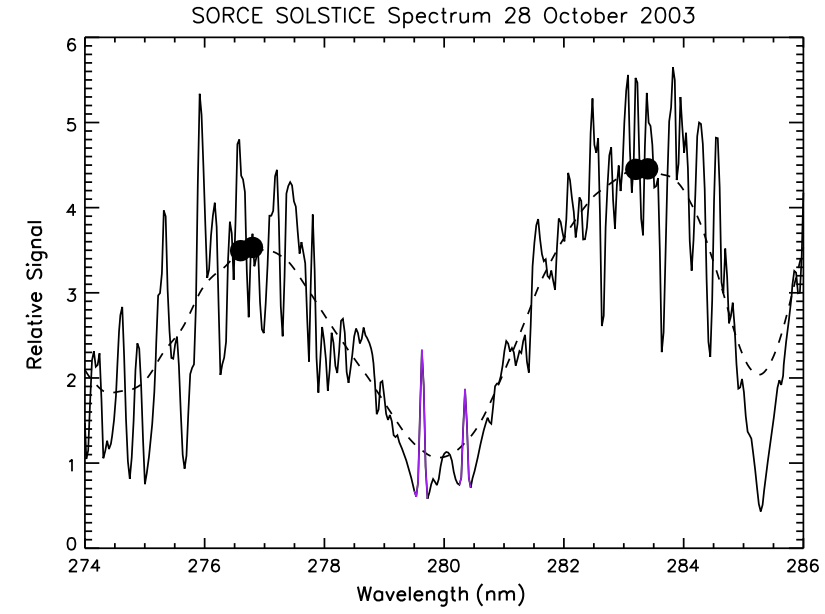

Fig. 2. The MgII doublet region as observed by SORCE SOLSTICE on 28 October 2003 . The $\mathrm{h} \& \mathrm{k}$ emission cores are highlighted in purple. The dashed line is the spectrum after convolving it with a $1.1 \mathrm{~nm}$ triangular bandpass. Black dots indicate the wing irradiance values used to calculate the index.

\subsubsection{NOAA 16 SBUV/2}

NOAA 16 has been in operation since 20 March 2001 and is still producing a MgII index measurement on a regular basis. The NOAA satellite operations web page (http://www.oso.noaa.gov) lists the diffuser status as "yellow" and the instrument's status is listed as "not nominal." The issue has to do with the orbital geometry and the reflection of sunlight into the instrument from the diffuser. The satellite is currently near the morning terminator, and is not always able to make a MgII index measurement (M. DeLand, private communication). As will be discussed in Section 3.2, this diffuser problem may or may not be influencing the data quality. The main topic of this paper is a comparison of the MgII index measurements through solar minimum, so the discrepancies starting in 2012 do not impact our conclusions.

\subsubsection{NOAA $17 \mathrm{SBUV/2}$}

NOAA 17 went into operation on 15 October 2002. and was decommissioned on 10 April 2013. However, the MgII index measurements ceased in September 2011. The orbit had drifted such that it was no longer possible to get sunlight into the instrument. The SBUV/2 instrument on NOAA 17 did not have any known anomalies when it stopped making solar measurements.

\subsubsection{NOAA $18 S B U V / 2$}

NOAA 18 became operational on 30 August 2005. After only a few years of operation, it developed a problem with the chopper motor. From May 2008 onwards, problems in operating the SBUV/2 instrument have limited the amount of data it has yielded. In December 2012, the chopper motor stalled, and no new solar data has been released since then.

\subsection{NASA}

Solar irradiance measurements near $280 \mathrm{~nm}$ from the SOLar Radiation and Climate Experiment (SORCE; Rottman 2005) 


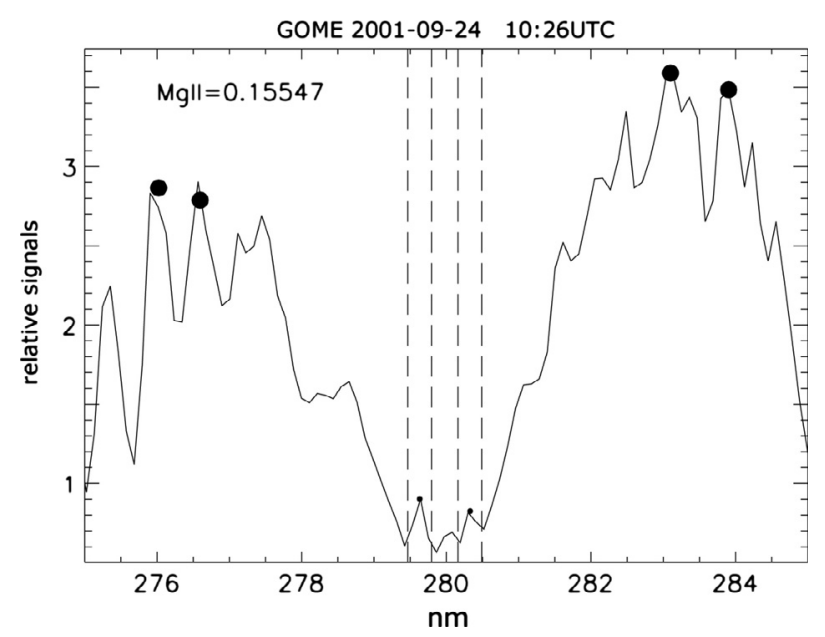

Fig. 3. The MgII doublet region as observed by GOME on 21 September 2001. Black dots indicate portions of the spectrum used to calculate the MgII index.

are the only observations available from a National Aeronautics and Space Administration (NASA) project. The SORCE mission includes two instruments that measure the relevant part of the solar spectrum, the SOLar-STellar Irradiance Comparison Experiment (SOLSTICE; McClintock et al. 2005) and the Solar Irradiance Monitor (SIM; Harder et al. 2005). Only the SOLSTICE data is published daily, so our study does not include SIM. The SIM data will likely become available to the public in the future, so it could be included in subsequent studies.

Figure 2 shows a spectrum from SORCE SOLSTICE. The spectral resolution of the instrument is $0.1 \mathrm{~nm}$, which is much higher than the other instruments used in this study. The complete description of the MgII index derived from the full-resolution spectrum is described in Snow et al. (2005). Briefly, the wing reference irradiance is determined by smoothing the spectrum to $1.1 \mathrm{~nm}$ with an SBUV-like triangular bandpass. The core irradiance is determined by fitting Gaussian profiles to the self-reversed emission lines. In Figure 2, the emission cores are highlighted in purple. The precision of the MgII index measurement from SOLSTICE was estimated to be $0.6 \%$.

The SOLSTICE experiment on SORCE actually consists of two entirely redundant instruments known as SOLSTICE A and SOLSTICE B. Each instrument has two channels, one to measure the far ultraviolet (FUV) and a second one to measure the middle ultraviolet (MUV). In normal operation, SOLSTICE A is primarily used to measure the MUV range, while SOLSTICE B monitors the FUV. The two instruments are intercompared in both channels at regular intervals to ensure consistency in their calibration. In the early part of the mission, this crosscalibration activity was performed once per week. Starting in the fall of 2009, the two SOLSTICEs started making simultaneous MUV observations once per day.

\subsection{ESA}

There are three European satellites which made MgII observations on a daily basis during the time period under study. Similar to the SBUV/2 instruments described earlier, the primary mission for these three instruments is to study the Earth backscattered solar spectral irradiance (SSI) to determine the atmospheric properties. Once per day, the incident ultraviolet SSI is measured using a diffuser plate to direct the sunlight straight into the instrument. These daily measurements are used to produce the MgII index used in this study. The data from GOME, SCIAMACHY, and GOME-2 have been combined into a single composite time series which is available from the University of Bremen at http://www.iup.uni-bremen.de/ gome/. For convenience, this composite will be referred to as the "Bremen" composite. In addition to scaling the datasets, there have been a number of additional corrections to the observations for this composite. A description of the corrections is included in Section 2.3.4.

\subsubsection{GOME}

The Global Ozone Monitoring Experiment (GOME) was launched in 1995 on the European Remote Sensing (ERS-2) satellite. The instrument and early observations are described in Weber et al. (1998). It continued to make observations until July 2011. Due to optical degradation in the harsh space environment, the signal-to-noise ratio degraded and the MgII index became very noisy toward the end of the data record. GOME's spectral resolution in the uv is $0.17 \mathrm{~nm}$, which is sufficient to partially resolve the MgII emission cores. Figure 3 shows a typical GOME spectrum around $280 \mathrm{~nm}$. The vertical dashed lines indicate the spectral range over which the spline interpolated core emissions have been integrated to obtain the numerator of the MgII index. The average wing values are determined by fitted parabolas, whose maxima are indicated as well in Figure 3. A complex etalon correction due to contaminants on the detector is applied to the solar irradiance before determining the index values (Weber et al. 1998). The etalon correction is monitored regularly and updated throughout the mission.

\subsubsection{SCIAMACHY}

The Scanning Imaging Absorption Spectrometer for Atmospheric CHartographY (SCIAMACHY) is a follow-up instrument with extended atmospheric observation capability that was launched on Envisat in March 2002 (Bovensmann et al. 2011). The UV spectral channels and solar measurement sequences over a diffuser plate are very similar to GOME. The spectral resolution is slightly lower at $0.2 \mathrm{~nm}$ but the calculation of the MgII index is very similar to that of GOME. The communication to Envisat was lost in April 2012.

\subsection{3. $G O M E-2$}

The GOME-2 aboard Metop A (Callies et al. 2000) was launched by EUMETSAT in late 2006. Near $280 \mathrm{~nm}$, the spectral resolution is about $0.3 \mathrm{~nm}$ which means that the emission cores are barely visible. Unlike for GOME and SCIAMACHY, a low spectral resolution MgII index definition according to Heath \& Schlesinger (1986) has been applied. The instrumental resolution was degraded using an 11-point (sampling every $0.11 \mathrm{~nm}$ ) triangle convolution before determining the average core and wing values of the MgII index. Figure 4 shows a typical MgII spectrum from GOME-2 at native spectral resolution and at about $1.2 \mathrm{~nm}$ spectral resolution. The solid points indicate the wing and core values used to calculate the core-to-wing ratio. GOME-2 continues operation through the present date. A follow-up instrument aboard Metop B was launched in 2012 and provides MgII data, but it has not been used here. 


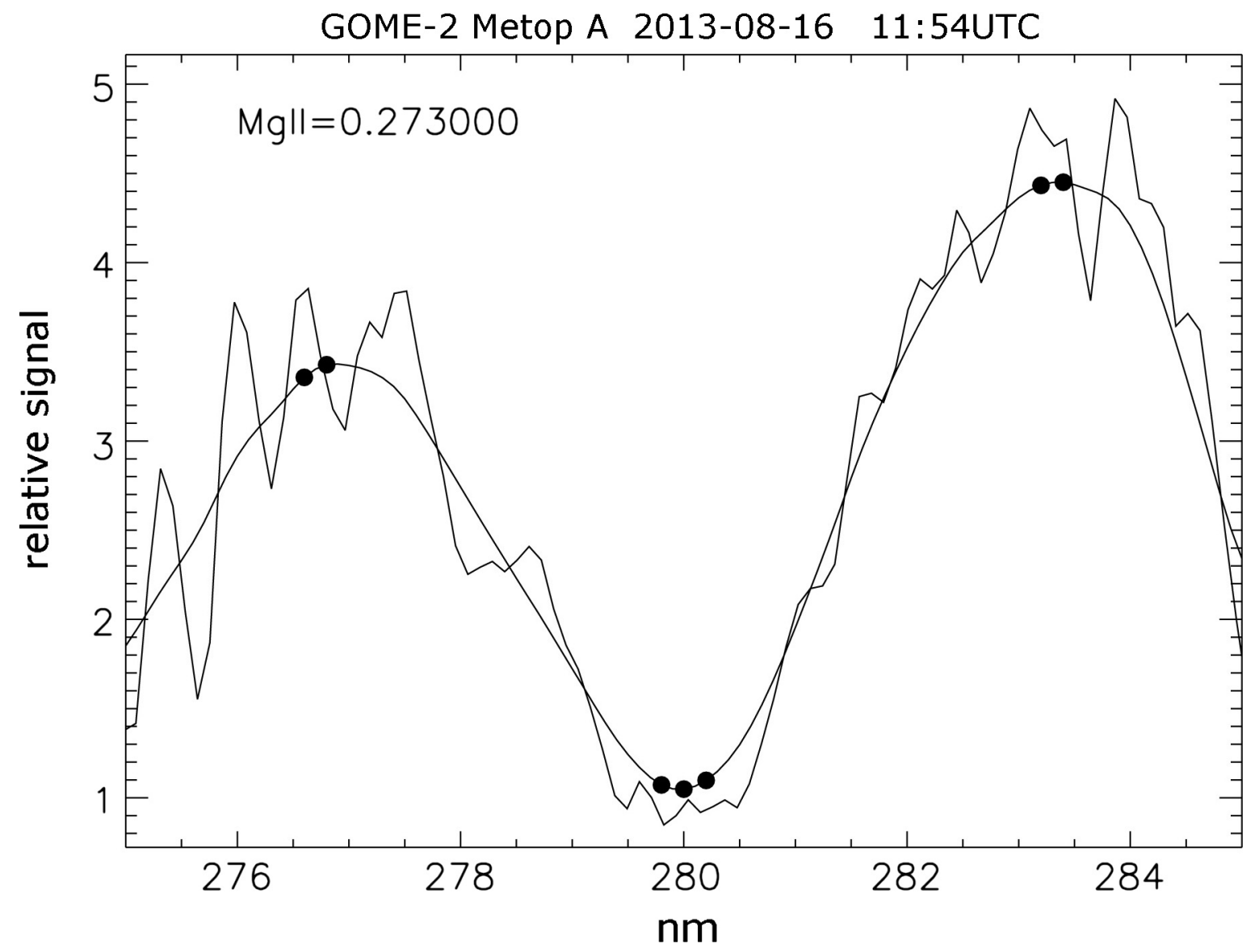

Fig. 4. The MgII doublet region observed by GOME-2.

\subsubsection{The Bremen composite}

Using the ESA MgII index data (GOME, SCIAMACHY, and GOME-2), a composite index has been created that also includes all available MgII index data from the SBUVs and both SOLSTICE instruments (UARS and SORCE). Until 1992, the base of the composite is the modified SBUV data from Viereck \& Puga (1999). From 1992 to 1995, it uses UARS SOLSTICE data. From 1995 to 2002 GOME is used, 2002-2008 uses SCIAMACHY, and from 2009 onwards the data come from GOME-2. All other concurrent available instruments have been successively used to fill data gaps. About 931 days with no MgII data available were filled with F10.7 cm flux data using a scaling period of about 200 days around each missing day. For the major period considered here, the Bremen composite is dominated by the European datasets. To match the various MgII indices with arbitrary units, a doubled linear scaling was applied for overlapping data by regressing index A vs. index B and index B vs. index A, and then using an average from both.

Both SCIAMACHY (January 2009) and GOME-2 (September 2009) had a major decontamination phase when the instruments were heated to remove contaminants and ice from the detector arrays to improve optical throughput. Both SCIAMACHY and GOME-2 show small discontinuities in the MgII index time series of a few tenths of a percent after the decontamination procedure. These jumps are several percent of the solar cycle variation, so they are significant corrections to the data. For this reason, both the GOME-2 and SCIAMACHY data were treated as separate time series before and after their respective decontamination procedures and separately scaled. The NOAA-16 data prior to January 2012 was used as a transfer standard and was scaled to SCIAMACHY before January 2009. Both GOME-2 time series before and after the decontamination were successively matched and the gaps filled with SCIAMACHY data after January 2009 and other available data.

\section{Data scaling}

The absolute scaling of the MgII index from a given instrument depends primarily on the spectral resolution. Low-resolution instruments such as the SBUV series generally just take ratios of irradiance samples, while instruments with higher resolution may use more complex algorithms. Users of the MgII index will typically want to have all of the measurements scaled to a common reference dataset. The standard method for determining the linear scaling factor is to merely plot one dataset against another during a period of overlapping measurements and fit a line to the result (Viereck \& Puga 1999; Viereck et al. 2004). For some datasets, the period of overlapping measurements may be fairly brief, but others may have many years of simultaneous daily measurements.

The interval of time used to determine the linear correlation between two datasets can have an influence on the long-term behavior of the composite. If the overlap takes place during a period of low solar activity, the uncertainty in the slope of the best fit line can be relatively larger than a fit that includes a wider range of index values. An instrumental drift in one dataset can propagate 


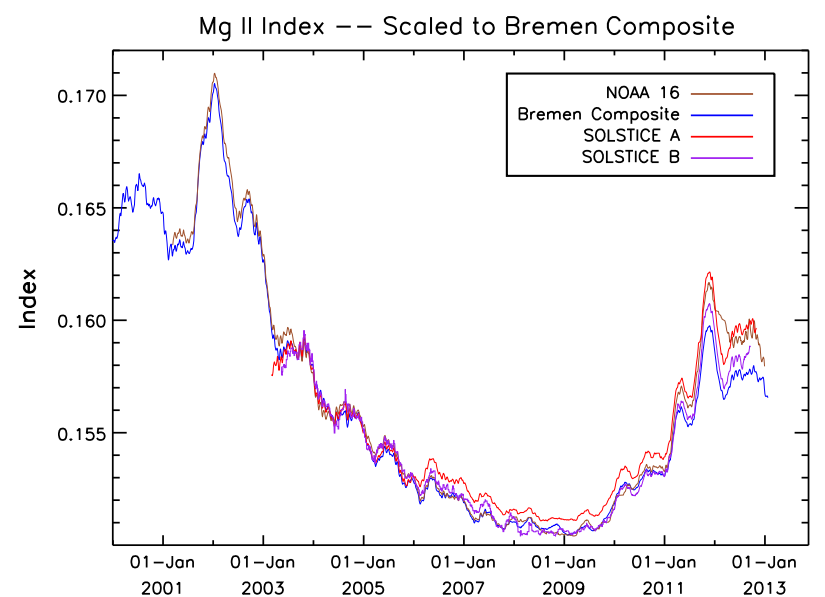

Fig. 5. Time series of MgII index observations scaled to the Bremen composite. The blue curve is the composite created from GOME, SCIAMACHY, and GOME-2 datasets. SORCE SOLSTICE A and B are the red and purple curves. The brown curve is the data from NOAA-16. All data has been smoothed over an 81-day window and scaled to match the Bremen composite during calendar year 2004.

into subsequent datasets if a long period of overlap is used. In order to study the relative changes in the MgII index datasets during solar minimum, we have chosen to use a time period during the descending phase of solar cycle 23, well before solar minimum. We have used the one-year time period from 1 January 2004 to 1 January 2005 to determine the scaling factors. The solar variability during this one-year period is about $42 \%$ of the full solar cycle range, so the uncertainty in the slope of the best fit line is not a significant source of uncertainty in the dataset comparison.

All of the datasets have been scaled to the Bremen composite. During this time period, i.e., solar minimum 23/24, the Bremen composite is dominated by data from SCIAMACHY and GOME-2. Figure 5 shows the Bremen composite, the observations from NOAA 16, and the observations from the two SOLSTICE instruments (A and B). In this figure, each dataset has been smoothed over three solar rotations (81 days) in order to highlight the long-term trends in the observations.

\subsection{Correction to SOLSTICE A}

What is clear from Figure 5 is that the measurements from SOLSTICE A diverge from the other datasets at the start of 2006. Although the MgII index is designed to be free of instrumental wavelength. Examples of instrumental artifacts that are not canceled out in the core-to-wing artifacts, it only cancels out multiplicative factors such as responsivity or degradation effects which are roughly linear with ratio would be a contribution from scattered light or uncorrected background counts. Some instrumental effects are very complex such as the etalon pattern in the GOME measurement (Weber et al. 1998).

The sudden deviation of the SOLSTICE A time series from all of the other instruments at the beginning of 2006 coincides with a known instrument anomaly. Part of the SOLSTICE design that allows it to observe both the Sun and bright stars with the same optics and detectors is that it has a mechanism to swap entrance apertures. The stellar aperture is a large $16 \mathrm{~mm}$ circle, while the solar aperture is a small $0.1 \mathrm{~mm}$ by $0.1 \mathrm{~mm}$ square. A bistable mechanism rotates a shaft to put

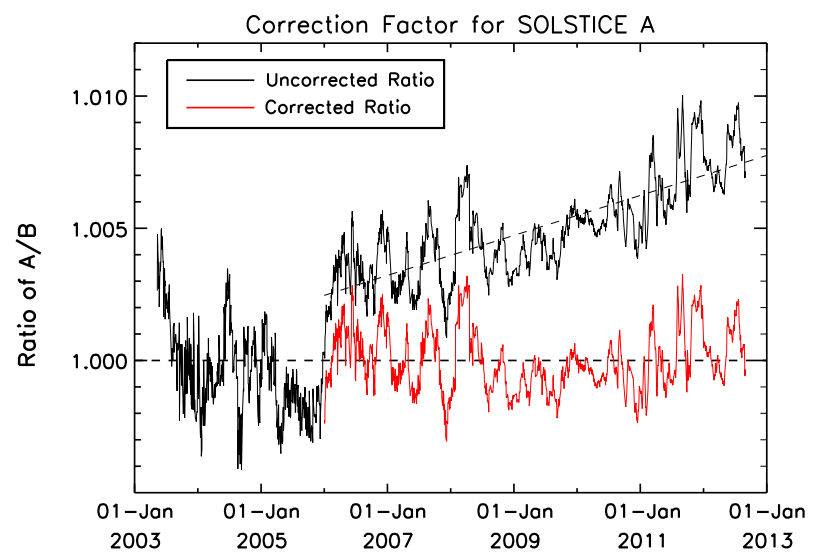

Fig. 6. Correction factor for SOLSTICE A data. This figure shows the ratio of daily average values for the MgII index measured by SOLSTICE A and SOLSTICE B. The ratio varies about 1 until the beginning of 2006 when there is a distinct discontinuity followed by a linear trend shown by the dashed line. The red curve shows the ratio between the two datasets after correction.

one of the two apertures into the optical path. In January 2006, as the spacecraft entered the sunlit portion of the orbit, commands were automatically sent to move the solar aperture back into place. However, the aperture mechanism did not move after the first few attempts. Eventually, the entrance aperture mechanism did operate and the solar slit was put in place. The operations team decided to leave the SOLSTICE A entrance aperture in place thereafter rather than risk having it stuck in stellar mode. Careful analysis over the following weeks showed that the entrance aperture had not seated in the exact spot that it had previously occupied. It is not clear how the change in the SOLSTICE A MgII index is the result of the entrance aperture anomaly, but the timing makes it unlikely to be a mere coincidence. Therefore, we adjust SOLSTICE A with the assumption that the SOLSTICE B measurement is correct, and SOLSTICE A is the anomalous measurement.

The ratio of SOLSTICE A to SOLSTICE B is shown in Figure 6. The black curve shows the ratio of the MgII index daily averages for the two instruments. This ratio is close to unity until the beginning of 2006. There is a sharp discontinuity and then the ratio has a non-zero slope from that point onwards. Without a physical understanding of the cause of this trend in the SOLSTICE A data relative to SOLSTICE B, we use a simple linear fit to the ratio to determine a correction factor. The linear fit is indicated by the dashed line. The ratio after correction by the linear fit is shown by the red curve.

In Snow et al. (2005), the precision of a single full-resolution MgII index measurement from SOLSTICE was estimated to be $0.6 \%$. The uncertainty of the mean of about ten observations in a day is about three times smaller than that, or close to $0.2 \%$. The day-to-day scatter in the ratio shown in Figure 6 is roughly consistent with these estimates. The mean of the ratio over the full SORCE mission after the linear correction has been applied is 0.9999 with a standard error of $0.144 \%$. For the time period before 2006, the mean and standard error of the ratio is 0.9996 and $0.166 \%$. Therefore, there is no justification for a more complex correction factor than a simple line. Both distributions (pre-anomaly and full mission with correction) are fairly Gaussian, so the use of normal statistics is reasonable. 


\subsection{Corrections after solar miniumum}

The deviation from the other datasets by SOLSTICE A during solar minimum was very clear and easily traced to an artifact in that instrument's time series. The divergence of the datasets in the spring of 2011 after the first large activity spike of solar cycle 24 is less easily understood. NOAA 16 started reporting a higher value (after scaling) than SOLSTICE B and it became much noisier. The instrument is very old, so it is possible that its solar measurement has degraded.

During this epoch, the Bremen composite is primarily using data from GOME-2. It started reporting a value lower than SOLSTICE B. This deviation will have to be understood in order to have confidence in the most recent values of any MgII index composite. Work on a grand composite is underway, and it will be described in a future publication.

\section{Discussion}

During the solar minimum between cycles 23 and 24, there were many instruments measuring the MgII core-to-wing ratio. This wealth of observational datasets has allowed us to uncover an instrumental trend in the measurements from SOLSTICE A. After this correction using observations from SOLSTICE B, the time series from SORCE is in good agreement with the Bremen composite. All the MgII measurements now show the same relative change in chromospheric activity during the decline of solar cycle 23 through the extended solar minimum.

There is still a discrepancy between the measurements during the rise in activity during cycle 24 , but further analysis of the datasets themselves will be required before a full composite can be constructed. Comparison between the different instruments will guide the creation of the new composite as much as possible, but the number of available datasets will be much fewer than we had during solar minimum. As described in the data catalog above, NOAA 17 and NOAA 18 have both been taken out of service. GOME and SCIAMACHY have also ceased to provide data, leaving only an aging NOAA 16 and a pair of GOME-2 instruments taking measurements going forwards. SORCE has suffered from degraded battery performance, and the last daily measurements of the MgII index from SOLSTICE were taken in July 2013. It is unlikely that SORCE SOLSTICE will ever resume daily measurements. The pair of GOME-2 instruments are in their prime mission, and the future GOES-R series instruments (Snow et al. 2009) will ensure that high-quality measurements of the MgII index will continue well into the future.

Acknowledgements. This work was supported by NASA Cooperative Agreement NNX13AI25A - Multisatellite Ultraviolet Solar Spectral Irradiance Composite (MUSSIC) - to the University of Colorado. MW acknowledges the partial financial support under grant agreement 313188 (SOLID) from the European Community's Seventh Framework Programme (FP7 2012).

\section{References}

Bovensmann, H., I. Aben, M. van Roozendael, S. Kühl, M. Gottwald, et al., SCIAMACHY's view of the changing Earth environment. In: Chapter 10 in SCIAMACHY - Exploring the Changing Earth's Atmosphere, edited by M., Gottwald and H. Bovensmann, Dordrecht, Springer, DOI: 10.1007/978-90-481-9896-2 10, 2011.

Callies, J., E. Corpaccioli, M. Eisinger, A. Hahne, and A. Lefebvre, GOME-2 - Metop's second generation sensor for operational ozone monitoring, ESA Bull., 102, 28-36, 2000.

Cebula, R.P., M.T. Deland, and B.M. Schlesinger, Estimates of solar variability using the Solar Backscatter Ultraviolet (SBUV) $2 \mathrm{Mg}$ II index from NOAA 9 satellite, J. Geophys. Res., 97, 1161311620, DOI: 10.1029/92JD00893, 1992.

DeLand, M.T., and R.P. Cebula, Composite Mg II solar activity index for solar cycles 21 and 22, J. Geophys. Res., 98, 1280912823, DOI: 10.1029/93JD00421, 1993.

Donnelly, R.F., The solar Mg II core-to-wing ratio from the NOAA9 satellite during the rise of solar cycle 22, Adv. Space Res., 8, 777780, DOI: 10.1016/0273-1177(88)90174-3, 1988.

Donnelly, R.F., Solar UV temporal variations during solar cycle 22 and the twentieth century. In: Climate Impact of Solar Variability, NASA CP-3086: 328-335, 1990.

Frederick, J.E., R.P. Cebula, and D.F. Heath, Instrument characterization for the detection of long-term changes in stratospheric ozone: an analysis of the SBUV/2 radiometer, J. Atmos. Oceanic Technol., 3, 472-480, 1986.

Harder, J., G. Lawrence, J. Fontenla, G. Rottman, and T. Woods, The spectral irradiance monitor: scientific requirements, instrument design, and operation modes, Sol. Phys., 230, 141-167, DOI: 10.1007/s11207-005-5007-5, 2005.

Heath, D.F., and B.M. Schlesinger, The Mg $280 \mathrm{~nm}$ doublet as a monitor of changes in the solar ultraviolet irradiance, J. Geophys. Res., 91, 8672-8682, DOI: 10.1029/JD091iD08p08672, 1986.

Lean, J.L., T.N. Woods, F.G. Eparvier, R.R. Meier, D.J. Strickland, J.T. Correira, and J.S. Evans, Solar extreme ultraviolet irradiance: present, past, and future, J. Geophys. Res., 116, A01102, DOI: 10.1029/2010JA015901, 2011.

McClintock, W.E., G.J. Rottman, and T.N. Woods, Solar-stellar irradiance comparison experiment II (SOLSTICE II): instrument concept and design, Sol. Phys., 230, 205-224, DOI: 10.1007/s11207-005-7432-x, 2005.

Rottman, G., The SORCE mission, Sol. Phys., 230, 7-25, DOI: 10.1007/s11207-005-8112-6, 2005.

Solomon, S.C., and L. Qian, Solar extreme-ultraviolet irradiance for general circulation models, J. Geophys. Res., 110, A10306, DOI: 10.1029/2005JA011160, 2005.

Snow, M., and W.E. McClintock, High time cadence solar magnesium II index monitor, Proc. SPIE, 5901, 354, DOI: 10.1117/12.617044, 2005.

Snow, M., W.E. McClintock, T.N. Woods, O.R. White, J.W. Harder, and G. Rottman, The magnesium II index from SORCE, Sol. Phys., 230, 325, DOI: 10.1007/s11207-005-6879-0, 2005.

Snow, M., W.E. McClintock, D. Crotser, and F.G. Eparvier, EUVS$\mathrm{C}$ : the measurement of the magnesium II index for GOES-R EXIS, Proc. SPIE, 7438, 1, DOI: 10.1117/12.828566, 2009.

Weber, M., J.P. Burrows, and R.P. Cebula, GOME solar uv/vis irradiance measurements between 1995 and 1997 - first results on proxy solar activity studies, Sol. Phys., 177, 63-77, 1998.

Viereck, R.A., and L.G. Puga, The NOAA Mg II core-to-wing solar index: construction of a 20-year time series of chromospheric variability from multiple satellites, J. Geophys. Res., 104, 999510005, DOI: 10.1029/1998JA900163, 1999.

Viereck, R.A., L.E. Floyd, P.C. Crane, T.N. Woods, B.G. Knapp, G. Rottman, M. Weber, L.C. Puga, and M.T. Deland, A composite Mg II index spanning from 1978 to 2003, Space Weather, 2, S10005, DOI: 10.1029/2004SW000084, 2004. Cite this article as: Snow M, Weber M, Machol J, Viereck R \& Richard E: Comparison of Magnesium II core-to-wing ratio
observations during solar minimum 23/24. J. Space Weather Space Clim., 2014, 4, A04. 